\title{
On the cohomology of sheaves $\mathscr{P} \& L$
}

by

\author{
NGUYEN VAN KHUE (Warszawa)
}

Abstract. Some resultis on the cohomology groups of sheaves $\mathscr{S}_{\varepsilon} I$ are given. Using those results we get the splitness of Dolbeault complexes of holomorphic Banach bundles at only positive dimension over Stein manifolds.

Introduction: Let $L$ be a complex locally convex space and let $L^{\prime}$ denote the strongly dual space of $L$. By $\mathscr{U}(L)$ we denote the set of all balanced convex neighbourhoods of zero in $L$. For every $U \in \mathscr{U}(L)$ let $p(U)$ denote the Minkowski functional of $U$ and let $L(U)$ be the completion of $L(U) / p(U) \stackrel{\text { def }}{=} L(U) / p(U)^{-1}(0)$ equipped with the norm $p(U)$.

A locally convex space $L$ is said to be $s$-nuclear (see [9]) if for every $U \in \mathscr{U}(L)$ there exists a $V \in \mathscr{U}(L)$ such that $V \subset U$ and the canonical $\operatorname{map} \omega(V, U): L(V) \rightarrow L(U)$ is $s$-nuclear, i.e., there exist sequences $\left\{\lambda_{j}\right\}$ $\subset \boldsymbol{R},\left\{u_{j}^{\prime}\right\} \subset L(V)^{\prime}$ and $\left\{v_{j}\right\} \subset L(U)$ such that

$$
\begin{aligned}
& \sup \left\{\left\|u_{i j}^{\prime}\right\|+\left\|\dot{b}_{j}\right\|: j=1,2, \ldots\right\}<\infty, \\
& \lambda_{1} \geqslant \lambda_{2} \geqslant \ldots>0 \\
& \sum_{j=1}^{\infty} \lambda_{j}^{p}<\infty \quad \text { for } \quad p>0
\end{aligned}
$$

and

$$
\omega(V, U) u=\sum_{j=1}^{\infty} \lambda_{j} u_{j}^{\prime}(u) v_{j} \quad \text { for } \quad u \in L(V) .
$$

Lot $(X, \mathcal{O})$ be an andytic space and $\mathscr{S}$ a coherent analytic sheaf on $X$. For every quasi-complete locally convex space $I$ consider the showif $\mathscr{S} \& L$ on $X$ generated by the presheaf

$$
U \mapsto \mathscr{S}(U) \& L \stackrel{\text { dot }}{=} \operatorname{HOM}_{s}\left(L_{c}^{\prime}, \mathscr{P}(U)\right)
$$

for every open set $U \subset X$, where $L_{c}^{\prime}$ denotes the space $L^{\prime}$ equipped with the compact-open topology and $\operatorname{HOM}_{s}\left(L_{o}^{\prime}, \mathscr{S}(U)\right)$ the space of all continuous linear maps trom $L_{0}^{\prime}$ into $\mathscr{S}(U)$ equipped with the topology of 
uniform convergence on all equicontinuous subsets of $L^{\prime}$. Since the presheaf $U_{\mapsto \mapsto \mathrm{HOM}_{\varepsilon}}\left(L_{c}^{\prime}, \mathscr{S}(U)\right)$ is a sheaf, we have

$$
H^{0}(U, \mathscr{S} \in L)=H^{0}(U, \mathscr{P}) \varepsilon L
$$

for every open set $U \subset X$, where we write $H^{0}(U, \mathscr{S})=\mathscr{S}(U)$.

Let us note that $H^{0}(U, \mathcal{O} \in L)$ is the space of holomorphie functions on $U$ with values in $L$, equipped with the compact-open topology [2].

The aim of this paper is to study the cohomology groups of sheaves $\mathscr{S} \varepsilon L$. In $\S 1$ we prove that if $X$ is an analytic space having a countables topology and $\mathscr{S}$ a coherent analytic sheaf on $X$ and if $H^{1}\left(X, \mathscr{S}_{\&} H^{\prime}\right)=0$ for every s-nuclear Fréchot space $Z{ }^{\prime}$, then $\mathscr{S}(X)$ is isomorphic to $C^{m}$ for some $m \leqslant \infty$. Moreover, we prove also that if $X$ is a Stein space, then $H^{q}(X, \mathscr{S} \& L)=0$ for every $q \geqslant 2$ and for every quasi-complete locally. convex space $L$. These results will be applied to the study of the splitness of Dolbeault complexes of holomorphic Banach bundles over complex manifolds in $\$ 2$.

Acknowledgments. The author wishes to express his gratitude to Professor W. Żelazko and Dr. E. Ligocka for helpful discussions during the preparation of the paper.

1. Cohology groups of sheaves $\mathscr{S}_{\&} L$. In this section we prove the following

THEOREM 1.1. (i) Let $X$ be an analytio spase having a oountable topology and $\mathscr{S}$ a coherent analytic sheaf on $X$. If $H^{1}\left(X, \mathscr{S}_{\&} \mathbb{H}^{\prime}\right)=0$ for every s-nuclear Fréchet space $F$, then $H^{0}(X, \mathscr{S})$ is isomorphio to $C^{m}$ for some $m \leqslant \infty$.

(ii) If $X$ is a compact analytio space and $\mathscr{S}$ a coherent analytio sheaf on $X$ such that $H^{q}(X, \mathscr{S})=0$ for some $q>0$, then $H^{\alpha}\left(X, \mathscr{S}_{\&} L\right)=0$ for every quasi-complete locally conves space $L$.

THEOREM 1.2. (i) If $X$ is an irreducible analytio space having a nonconstant holomorphio function and if $F$ is a Fréchet space whioh does not admit a continuous norm, then $H^{1}\left(X, O \& \& F^{\prime}\right) \neq 0$.

(ii) If $X$ is a Stein space and $\mathscr{S}$ a coherent analytio sheaf on $X$, then

$$
H^{\alpha}\left(X, \mathscr{S}_{\varepsilon} X\right)=0
$$

for every $q \geqslant 2$ and for every quasi-complete looally conveo spaoe $L$.

The proof of Theorem 1.1 is based on the following

Propostrion 1.3. Let $E$ be a subspace of a Frechet space If. If $t$ is s-nuclear, then there exist an s-nuolear Frechet space $\tilde{\mathbb{B}}$ and continuous linear maps $e: E \rightarrow \tilde{E}$ and $h: F \rightarrow \tilde{\mathbb{E}}$ such that $h \mid \mathbb{E}=e$ and $e$ is an embedding.
We need the following.

Liemma 1.4. For every s-nuclear map $T$ from a Banach space $\dot{A}$ into a Banach space $B$ there exist s-nuclear maps $P$ and $Q$ between Banach spaces such that $T=Q P$.

Proof. Since $T$ is $s$-nuclear, there exist sequences $\left\{\lambda_{j}\right\} \subset \boldsymbol{R},\left\{u_{j}^{\prime}\right\} \subset A^{\prime}$ and $\left\{v_{j}\right\} \subset B$ satisfying condition (S) and such that

$$
T u=\sum_{j=1}^{\infty} \lambda_{j} u_{j}^{\prime}(u) v_{j} \quad \text { for } \quad u \in A .
$$

For $u \in A$ and $\xi=\left(\xi_{j}\right) \in l^{1}$, putting

$$
P u=\sum_{j=1}^{\infty} \sqrt{\lambda_{j}} u_{j}^{\prime}(u) e_{j}, \quad Q \xi=\sum_{j=1}^{\infty} \sqrt{\lambda_{j}} \xi_{j} v_{j}
$$

where $\left\{e_{j}\right\}$ denotes the canonical basis of $l^{1}$, we get $s$-nuclear maps $P$ and $Q$ such that $T=Q P$.

Limara 1.5. Every s-nuclear map $T$ from a subspace of a Banach space into a Banach space oan be extended to an s-nuolear map.

Proof. The lemma follows from the Hahn-Banach theorem.

Proof of Proposition 1.3. Let $\left\{J_{n}\right\}$ be a decreasing sequence of balanced convex neighbourhoods of zero in $F$ such that

(a) $\left\{U_{n} \cap E\right\}$ forms a basis of neighbourhoods of zero in $E$.

(b) The canonical maps $\eta_{n+1}^{n}=\omega\left(U_{n+1} \cap E, U_{n} \cap E\right): E_{n+1} \rightarrow E_{n}$ are $s$-nuclear, where $\mathbb{E}_{n}=\mathbb{E}\left(\bar{U}_{n} \cap E\right)$. By Lemma 1.5 for each $n \geqslant 1$ there exists an 8 -nuclear map $\tilde{\eta}_{n+1}^{n}: \mathbb{F}_{n+1} \rightarrow E_{n}$ such that $\tilde{\eta}_{n+1}^{n} \mid \mathbb{E}_{n+1}=\eta_{n+1}^{n}$, where $F_{n}=F\left(D_{n}\right)$. Applying Lemma 1.4 to $\tilde{\eta}_{n+1}^{n}$ we get $s$-nuclear maps $P_{j}^{n}$ and $Q_{j}^{n}$ such that

$$
\tilde{\eta}_{n+1}^{n}=Q_{1}^{n} P_{1}^{n}, \ldots ; P_{j}^{n}=Q_{j+1}^{n} P_{j+1}^{n}, \ldots
$$

Let $\hat{D}_{n}$ denote the completion of $F_{n} / p_{n}$ equipped with the norm

$$
p_{n}(\bar{u})=\left\|\tilde{\eta}_{n}^{n-1} u\right\|+\left\|P_{n-2}^{1} \omega_{n}^{2} u\right\|+\ldots+\left\|P_{1}^{n-2} \omega_{n}^{n-1} u\right\|,
$$

where $\omega_{n+1}^{n}=\omega\left(U_{n+1}, U_{n}\right)$.

By (1.3) the map $\omega_{n+1}^{n}$ induces naturally a continuous linear map $\tilde{\omega}_{n+1}^{n}$ : $\tilde{\mathbb{E}}_{n+1} \rightarrow \tilde{\mathbb{H}}_{n}$. Putting

$$
\tilde{\mathbb{B}}=\lim _{\leftarrow}\left\{\mathbb{W}_{n}, \tilde{\omega}_{n}^{m}\right\}, \quad h=\lim _{\leftarrow} h_{n},
$$

where $h_{n}: W_{n} \rightarrow \tilde{\mathbb{H}}_{n}$ denotes the canonical map, we get a Fréchet space $\tilde{E}$ and a continuous linear map $h: F \rightarrow \tilde{E}$ such that $e=h \mid \mathbb{B}$ is an embedding.

To tinish the proof it suffices to check that $\tilde{\omega}_{n+1}^{n-1}$ is $s$-nuclear for $n \geqslant 2$. For every $n \geqslant 2$, by (1.2) and (1.3) we can define a continuous 
linear map

$$
\alpha_{n+1}: \tilde{E}_{n+1} \rightarrow \operatorname{Im} \tilde{\eta}_{n}^{n-1} \oplus \operatorname{Im} P_{n-2}^{1} \oplus \ldots \oplus \operatorname{Im} P_{1}^{n-2}
$$

by the formula

$$
\alpha_{n+1}(\bar{u})=\left\{\tilde{\eta}_{n}^{n-1} \omega_{n+1}^{n} u, Q_{n-1}^{1} P_{n-1}^{1} \omega_{n+1}^{2} u, \ldots, Q_{2}^{n-2} P_{2}^{n-2} \omega_{n+1}^{n-1} u\right\} .
$$

Since $\alpha_{n+1}=\gamma_{n} \tilde{\omega}_{n+1}^{n}$, where $\gamma_{n}$ denotes the embedding of $\tilde{\boldsymbol{B}}_{n}$ into $\operatorname{Im} \tilde{\eta}_{n}^{n-1} \oplus$ $\oplus \operatorname{Im} P_{n-2}^{1} \oplus \ldots \oplus \operatorname{Im} P_{1}^{n-2}$ given by the formula

$$
\gamma_{n}(\bar{u})=\left\{\tilde{\eta}_{n}^{n-1} u, P_{n-2}^{1} \omega_{n}^{2} u, \ldots, P_{1}^{n-2} \omega_{1}^{n-2} u\right\}
$$

and since $Q_{j}^{n}$ are 8 -nuclear, it follows that $a_{n+1}$ is $s$-nuclear. Thus, by the following lemma, we infer that $\tilde{\omega}_{n+1}^{n-1}$ is $s$-nuclear.

LEMCMA 1.6. Let $\alpha: A \rightarrow B$ and $\beta: B \rightarrow 0$ be continuous linear maps such that ea is s-nuolear for some embedding $e: B \hookrightarrow \tilde{B}$ and $\beta$ is quasi-nuclear. Then $\beta \alpha$ is s-nuclear.

Proof. Since $\beta$ is quasi-nuclear, $\beta$ is represented in the form $\beta=\eta \gamma$, whero $\gamma: B \rightarrow l^{\infty}$ and $\eta: l^{\infty} \rightarrow C$ are continuous linear maps [9]. By the Hahn-Banach theorem there exists a continuous linear map $\tilde{\boldsymbol{\gamma}}: \tilde{\boldsymbol{B}} \rightarrow l^{\infty}$ such that $\tilde{\gamma} e=\gamma$. Since $e \alpha$ is $s$-nuclear, so is $\tilde{\gamma} e a$. Hence $\beta \alpha=\eta \tilde{\gamma} e \alpha$ is $s$-nuclear.

LEMra 1.7. Let F be a Fréchet-Montel space and let there exist a continuous linear map $P$ from $E=\prod_{j=1}^{\infty} B_{j}$, where $B_{j}$ are Banach spaces, onto F. Then $F \cong C^{m}$ for some $m \leqslant \infty$.

Proof. Let $G_{n}=\prod_{j=1}^{n} U_{j} \times \prod_{j>n} B_{j}$, where $U_{j}=\left\{u \in B_{j}:\|u\|<1\right\}$. Sinee $P$ is open, it follows that for every $n$, the formula

$$
P_{n}\left(\left\{u_{1}, \ldots, u_{n}\right\}\right)=\pi\left(P G_{h}\right) P\left(\left\{u_{1}, \ldots, u_{n}, 0, \ldots\right\}\right)
$$

defines a continuous linear open map from $E / p\left(G_{n}\right)=\prod_{j=1}^{n} B_{j}$ onto $F / p\left(P G_{n}\right)$, where $\pi\left(P G_{n}\right)$ denotes the canonical map from $F$ onto $F / P\left(P G_{n}\right)$. By hypothesis, $F$ is Montel, it follows that $P_{n}$ is compact. Honco $\operatorname{dim} F\left(P G_{n}\right)$ $<\infty$. On the other hand, since $\left\{P G_{n} / m\right\}_{n, m=1}^{\infty}$ forms a basis of neighbourhoods of zero in $F$, it follows that $F$ is isomorphic to $C^{m}$ for somo $m \leqslant \infty$.

LEMna 1.8. Let $X$ be an analytio spaoe and $I F$ a Freohet spaoe. Then for every $f \in\left(\mathcal{O} \in \mathbb{F}^{\prime \prime}\right)$ there exist $U \in \mathcal{U}\left(F^{\prime}\right)$ and $\tilde{f} \in\left(\mathcal{O} \in \mathbb{F}(U)^{\prime}\right)$ s sich that $f=\pi(U)^{\prime} \tilde{f}$.

Proof. Since a map $f$ from an analytic subset $V$ of an open set $G$ in $C^{n}$ into a quasi-complete locally convex space $L$ is holomorphic if and only if $f$ can be extended to a holomorphic map on a neighbourhood of every point $z \in V$ in $G$ [2], we can assume without loss of generality that $X$ is an open set in $C^{n}$ for some $n$. Let $f \in\left(\mathcal{O} \in F^{\prime}\right), z \in X$. Take a holomorphic map $\bar{f}$ from a neighbourhood $G$ of $z$ in $X$ into $L$ such that $\tilde{f}_{z}=f$ and $\tilde{f}(G)$ is bounded. Since $F$ is Fréchet, $\tilde{f}(G)$ is contained and bounded in $F(U)^{\prime}$ for some $U \in \mathscr{U}\left(W^{\prime}\right)$. Then, by the Cauchy integral formula, we infer that $\tilde{f}: G \rightarrow F^{\prime}(U)^{\prime}$ is holomorphic. Obviously $f=\left[\pi(U)^{\prime} \tilde{f}\right]_{x}$

Liencma 1.9. Let $X$ be a paraoompact analytio space and $\mathscr{S}$ a coherent analytio sheaf on $X$. Then $H^{0}(X, \mathscr{S})$ is s-nuolear.

Proof. (a) First we observe that subspaces and quotient spaces of 8 -nuclear spaces are 8 -nuclear.

(b) From (a) by the Oartan Theorem B [6] and by the paracompactness of $X$ wo can assume that $X$ is an analytic set in an open polydisc

$$
\Delta^{n}=\Delta_{1}^{n}=\left\{z=\left(z_{1}, \ldots, z_{n}\right) \in C^{n}: \max \left|z_{j}\right|<1\right\}
$$

in $\boldsymbol{C}^{n}$ for some $n$ and $S^{P}=\mathcal{O}$.

(c) Since the restriction map $\mathcal{O}\left(\Delta^{n}\right) \rightarrow \mathcal{O}(X)$ is surjective [6], it suffices to check that $\mathcal{O}\left(\Delta^{n}\right)$ is $s$-nuclear.

(d) Let $0<\varepsilon<1$ and lot $0<\delta<\varepsilon$. By the Taylor expansion at zero of overy element $f \in \mathcal{O}\left(\Delta^{n}\right)$, the canonical map

$$
\omega(\varepsilon, \delta): \mathcal{O}\left(\Delta^{n}\right)\left(U_{s}\right) \rightarrow \mathcal{O}\left(\Delta^{n}\right)\left(U_{\delta}\right)
$$

is represented in the form

$$
\omega(\varepsilon, \delta) f=\sum_{\alpha}(\delta / \varepsilon)^{|\alpha|} u_{\alpha}^{\prime}(f) v_{\alpha}
$$

where

$$
\begin{aligned}
& U_{a}=\left\{f \in \mathcal{O}\left(\Delta^{n}\right): \sup \left\{|f(z)|: z \in \bar{\Delta}_{\bullet}^{n}\right\} \leqslant 1\right\}, \\
& a=\left(\alpha_{1}, \ldots, \alpha_{n}\right), \quad|a|=\alpha_{1}+\ldots+\alpha_{n} \\
& u_{\alpha}^{\prime} \in\left[\mathbb{O}\left(\Delta^{n}\right)\left(U_{\theta}\right)\right]^{\prime} \text {, } \\
& u_{\alpha}^{\prime}(f)=\varepsilon^{|\alpha|} /(2 \pi i)^{n} \int_{\left|\xi_{j}\right|=\varepsilon} \frac{f(\xi) d \xi_{1} \ldots d \xi_{n}}{\xi_{1}^{a_{1}+1} \ldots \xi_{n}^{a_{n}+1}}, \\
& v_{\alpha} \in \mathcal{O}\left(A^{n}\right)\left(U_{\delta}\right), \quad v_{\alpha}(z)=1 / \delta^{|\alpha|} z_{1}^{\alpha_{1}} \ldots z_{n}^{a_{n}}, \quad z \in \Delta_{\delta}^{n} .
\end{aligned}
$$

Since $\sum_{a}(\delta / s)^{p|a|}<\infty$ for every $p>0$ and $\sup \left\{\left\|u_{a}^{\prime}\right\|+\left\|v_{a}\right\|\right\}<\infty$, it follows that $\omega(\varepsilon, \delta)$ is 8 -nuolear.

Proof of Theorem 1.1. (i). By Lemma 1.9 there exists a decreasing basis of balanced convex neighbourhoods of zero in $B=H^{0}(X, \mathscr{S})$, $\left\{U_{n}\right\}$, such that the maps $\eta_{n+1}^{n}=\omega\left(U_{n+1}, U_{n}\right): E_{n+1} \rightarrow E_{n}$ are $s$-nuclear. Put $F=\prod_{j=1}^{\infty} E_{j}$. Applying Proposition 1.3 to the canonical embedding 
$e: \boldsymbol{E} \rightarrow F, e(u)=\left\{\boldsymbol{\pi}\left(\nabla_{n}\right) u\right\}$, we get an $s$-nuclear Fréchet space $\tilde{\boldsymbol{E}}$ and continuous linear maps $\tilde{e}: E \rightarrow \tilde{\mathbb{E}}, h: F \rightarrow \tilde{\mathbb{E}}$ such that $\tilde{e}$ is an embedding and $h e=\tilde{e}$. Since $\tilde{\mathbb{E}} / \operatorname{Im} \tilde{e}$ is Fréchet-Montel, the sequence

$$
0 \rightarrow(\tilde{\mathbb{E}} / \operatorname{Im} \tilde{e})^{\prime} \rightarrow \tilde{\mathbb{E}}^{\prime} \stackrel{\tilde{e}^{\prime}}{\rightarrow} E^{\prime} \rightarrow 0
$$

is topologically exact. Hence the sequence

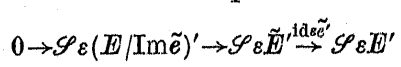

is exact. We prove that the map id $\varepsilon \tilde{e}^{\prime}$ is surjective. Let $z \in X$ and $f \in\left(\mathscr{S}_{\&} D^{\prime}\right)_{z}$. Take a surjective morphism $\mu: \mathscr{O}^{p}|G \rightarrow \mathscr{S}| G$ and $\tilde{f} \in H^{0}\left(G, \mathscr{S}_{\varepsilon} E^{\prime}\right)$ inducing $f$, where $G$ is a Stein neighbourhood of $z$ in $X$. Let $V$ bo a relatively compact neighbourhood of $z$ in $G$. Then there exists $W \in \mathscr{U}\left(\mathcal{O}^{p}(G)\right)$ such that $\mathcal{O}^{p}(G)(W)$ is isomorphic to a Hilbert space and $g|V=\pi(W) g| V$ for all $g \in \mathcal{O}^{p}(G)$. Since the map $\hat{\mu}_{G}: H^{0}\left(G, \mathcal{O}^{D}\right) \rightarrow H^{0}(G, \mathscr{S})$ induced by $\mu$ is surjective [6], $\hat{\mu}_{G}$ induces a continuous linear map $\gamma$ from $\mathcal{O}^{p}(G)(W)$ onto $H^{0}(G, \mathscr{S})(\tilde{W})$, where $\tilde{W}=\hat{\mu}_{\theta}(W)$. Thus there exists a continuous linear map $\beta: \mathbb{E} \rightarrow \mathcal{O}^{p}(V)$ such that $\hat{\mu}_{V} \beta=\tilde{f} \mid V$. By Lemma 1.8 we can assume that $\beta \in \mathcal{O}^{p}(V) \varepsilon \mathbb{E}\left(U_{0}\right)^{\prime}$ for some $U \in \mathscr{U}(\tilde{\mathbb{E}})$, where $U_{0}=\tilde{e}^{-1}(U)$. On the other hand, since the restriction map $\tilde{E}(U)^{\prime} \rightarrow[\operatorname{Im} \tilde{e}(U \cap \operatorname{Im} \tilde{e})]^{\prime}$ is surjective, there exists $\eta \in \mathscr{O}^{p}(V) \varepsilon \tilde{\mathbb{B}}^{\prime}$ such that (id. $\left.\varepsilon \tilde{e}^{\prime}\right) \eta=\beta$ [4]. Obviously

$$
\alpha=\left(\hat{\mu}_{V} \varepsilon \mathrm{id}\right) \eta \in H^{0}\left(V, \mathscr{S} \varepsilon \tilde{E}^{\prime}\right) \text { and } \quad\left[\left(\mathrm{id} \varepsilon e^{\prime}\right) \alpha\right]_{z}=f .
$$

Since $\tilde{\mathbb{E}} / \operatorname{Im} \tilde{\boldsymbol{e}}$ is $s$-nuclear, $H^{\mathrm{1}}\left(X, \mathscr{S}_{\varepsilon}(\tilde{\mathbb{E}} / \operatorname{Im} \tilde{\boldsymbol{e}})^{\prime}\right)=0$. Thus the map id $\varepsilon \tilde{e}^{\prime}: H^{0}\left(X, \dot{\mathscr{S}}_{\varepsilon} \tilde{E}^{\prime}\right) \equiv \operatorname{HOM}_{\triangleleft}(\tilde{E}, \mathscr{P}(X)) \rightarrow H^{0}\left(X, \mathscr{S}_{\varepsilon} E^{\prime}\right)=\operatorname{HOM}_{\odot}(L, \mathscr{S}(X))$ is surjective. Hence there exists an element $\theta \in \operatorname{FOM}_{\theta}(\tilde{\mathbb{E}}, \mathscr{S}(X))$ such that $\theta \tilde{e} \sigma=\sigma$ for all $\sigma \in H^{0}(X, \mathscr{S})$. Setting $P=\theta h$, we get a continuous linear map from $F$ onto $H^{0}(X, \mathscr{S})$. Since $H^{0}(X, \mathscr{S})$ is Fréchet-Montel, by Lemma $1.7 H^{0}(\boldsymbol{X}, \mathscr{S})$ is isomorphic to $\boldsymbol{C}^{m}$ for somo $m \leqslant \infty$. This completes the proof of (i).

(ii). By $\mathscr{B}(L)$ we demote the set of all bounded baluneed convex subsets of $L$. Let $K \in \mathscr{B}(L)$ and let $L(K)$ denote the Banach spaces spanned by $K$. This space is equipped with the norm $\varrho_{K}$, where $\varrho_{K}$ is the Minkowski functional of $K$ on $L(K)$. It is known [2] that

$$
\text { (1.4) } \quad\left(\mathscr{S}_{\varepsilon} L\right)_{s}=U\left\{\left(\mathscr{S}_{\varepsilon} L(K)\right)_{z}: K \in \mathscr{S}(L)\right\} \quad \text { for } \quad \approx \in X \text {. }
$$

Let $\omega \in H^{\alpha}\left(X, \mathscr{S}_{\varepsilon} L\right)$. Since $H^{q}\left(X, \mathscr{P}_{\varepsilon} L\right)=\lim H^{\alpha}\left(\mathscr{U}, \mathscr{S}_{\varepsilon} L\right)$, by the compactness of $X$ and by (1.4) we find an $K \in \overrightarrow{\mathscr{B}}(L)$ such that $\omega$ is induced by some element $\omega(K) \in Z^{q}\left(\mathscr{U}, \mathscr{S}_{\varepsilon} L(K)\right)$, where $\mathscr{U}$ is a Stein open covoring of $X$ and $Z^{q}\left(\mathscr{U}, \mathscr{S}_{\varepsilon} L(K)\right)=\operatorname{Ker} \delta^{\alpha}, \delta^{q}=\delta^{q}\left(\mathscr{U}, \mathscr{S}_{\varepsilon} L(K)\right): \partial^{\alpha}\left(\mathscr{U}, \mathscr{S}_{\varepsilon} L(K)\right)$ $\rightarrow C^{q-1}\left(\mathscr{U}, \mathscr{S}_{\varepsilon} L(K)\right)$ are coboundary maps and $\theta^{q}\left(\mathscr{U}, \mathscr{S}_{8} L(K)\right)$ aro oquipped with the product topology. Since $\mathscr{O}$ is a Leray covering of $X$, by hypo- thesis the map $\delta^{\alpha-1}: \mathscr{C}^{\alpha-1}(\mathscr{U}, \mathscr{P}) \rightarrow Z^{\alpha}(\mathscr{U}, \mathscr{S})$ is surjective. Then, by the nueleurity of $O^{\alpha-1}(\mathscr{U}, \mathscr{S})$ it follows that the map

$$
\delta^{\alpha-1}\left(\mathscr{U}, \mathscr{S}_{\varepsilon} L(K)\right): \sigma^{\alpha-1}\left(\mathscr{U}, \mathscr{S}_{\varepsilon} L(K)\right) \rightarrow Z^{q}\left(\mathscr{U}, \mathscr{S}_{\varepsilon} L(K)\right)
$$

is surjective. Trenee $\omega(K)=\delta^{q-1} \tilde{\omega}$ for some $\tilde{\omega} \in O^{q-1}\left(\mathscr{U}, \mathscr{L}_{\varepsilon} L(K)\right)$. Thus $\omega=0$ and (ii) is proved.

Troof of Theorem 1.2. (i): Let $H^{1}\left(X, O_{\varepsilon} \mathbb{H}^{\prime \prime}\right)=0$, where $F$ is some Frechet space which does not admit a continuous norm. By a theorem of Bessaga and Polczynski [1.], there exists a complemented subspace of $F$ which is isomorphic to $C^{\infty}$. Hence $H^{1}\left(X, O_{B} C^{\infty \prime}\right)=0$.

Let $f$ bo a non-constant holomorphic function on $X$. Put $a=\sup \{|f(z)|$ : $z \in X\}$. By the irroducibility of $X$ wo can assume that $a=\infty$ since if $a<\infty$, wo replace $f$ by the function $(r-f(z))^{-1}$, where $|r|=a$ and $r=\lim f\left(x_{n}\right)$ for as sequence $\left\{x_{n}\right\} \subset X$. Take a sequenco $\left\{z_{n}\right\} \subset R(X)$, where $R(X)$ ilenotes the regular part of $X$ such that $\left|f\left(z_{n}\right)\right| \rightarrow \infty$ and $f\left(z_{k}\right)$ $\neq f\left(\pi_{j}\right)$ for $k \neq j$. Select $p \in \mathcal{O}(C)$ such that $\varphi^{-1}(0)=\left\{f\left(z_{n}\right)\right\}_{n=1}^{\infty}$. Put $V=(p f)^{-1}(0)=\bigcup_{j=1}^{\infty} V_{j}$ where $V_{j}=f^{-1}\left(f\left(z_{j}\right)\right)$. Since $\left\{f\left(z_{j}\right)\right\}_{j=1}^{\infty}$ is discrete and since $f$ is continuous, there exists an open covering $\mathscr{U}=\left\{U_{j}\right\}_{j=0}^{\infty}$ of $X$ such that

$$
\begin{aligned}
& V_{j} \subset V_{j} \text { for } j \geqslant 1, \\
& U_{i} \cap U_{j} \neq \emptyset \quad \text { for } i \neq j, i, j \geqslant 1 \text { and } V \cap U_{0}=\emptyset .
\end{aligned}
$$

Put $f_{i j}=0$ for $i, j \geqslant 1$ and $f_{0 j}=-f_{j 0}=e_{j} / \varphi_{j}$ for $j \geqslant 0$, where $e_{0}=0$, $e_{j}=(\underbrace{0, \ldots, 0,1}), j \geqslant 1$. Obviously $\left\{f_{i j}\right\}, Z^{1}\left(\mathscr{C}, O_{\varepsilon} C^{\infty \prime}\right)$. Since $H^{1}\left(X, O_{\varepsilon} C^{\infty \prime}\right)$ $=0$, we can assume that $f_{i j}=f_{i}-f_{j}$, where $f_{i} \in H^{0}\left(O_{i}, O_{\varepsilon} C^{\infty \prime}\right)$ and thus we have.

$$
e_{i}+\varphi \rho f f_{i}=e_{j}+\varphi_{i} f_{j} \quad \text { on } \quad U_{i} \cap U_{j} \quad \text { for } \quad i, j \geqslant 0 .
$$

Thus the formula

$$
\tilde{f}(z)=e_{j}+q f f_{j}(z) \text { for } z \in U_{j}
$$

define in olement $\tilde{f} \in H^{\prime \prime}\left(X, O_{B} C^{\infty}\right)$ such that $\tilde{f}\left(V_{j}\right)=e_{j}$ for $j \geqslant 1$. Take $z_{0} \in R(X)$ and a meighibourhood $G$ of $z_{0}$ such that $\tilde{f}(G)$ is bounded. Then

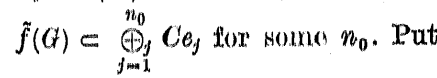

$$
\tilde{G}=\left\{z \in R(X): \exists G_{z} \ni z: \tilde{f}\left(G_{z}\right) \subset \bigoplus_{j=1}^{n_{0}} C e_{j}\right\} .
$$

Note thut $\tilde{G}$ is non-empty and open. Let $z \in \overline{\tilde{G}} \cap R(X)$ and let $G_{z}$ be at connected neighbourhood of $z$ in $R(X)$ such that $\tilde{f}\left(G_{z}\right) \subset \underset{j=1}{\oplus} C e_{j}$ for some $p$. 
Since $\tilde{f} \mid G_{z}: G_{z} \rightarrow \bigoplus_{j=1}^{n_{0}+p} C e_{j}$ is holomorphic, $\tilde{f}\left(G_{z} \cap \tilde{G}\right) \subset \bigoplus_{j=1}^{n_{0}} C e_{j}$ and $G_{z} \cap \tilde{G}$ is non-empty open in $G_{z}$, by the connectedness of $G_{z}^{j=1}$ it follows that $\tilde{f}\left(G_{z}\right)$ $\subset \bigoplus_{j=1}^{n_{0}} C e_{j}$. Thus $z \in \tilde{G}$ and hence $\tilde{G}$ is closed in $R(X)$. Since $X$ is irreducible, $R(X)$ is connected. Hence $\tilde{G}=R(X)$ and thus $\tilde{f}(R(X)) \subset \underset{j=1}{n_{0}} \boldsymbol{C} e_{j}$. This is impossible since $z_{n_{0}+1} \in R(X)$ and $\tilde{f}\left(z_{n_{0}+1}\right) \notin \oplus{ }_{\oplus}^{n_{0}} C e_{j}$. Thus (i) is proved.

(ii): Let $X, \mathscr{S}$ and $L$ be as in (ii) and $q \geqslant 2$. We prove that $H^{\alpha}\left(X, \mathscr{S}_{\varepsilon} L\right)$ $=0$. We write $X=\bigcup_{n=1}^{\infty} W_{n}$, where $W_{n}$ are relatively compact sets in
$X$ such that

$$
W_{n} \subset \hat{W}_{n} \subset W_{n+1} \quad \text { for } \quad n \geqslant 1 .
$$

It is known [2] (Theorem $B^{*}$, p. 338) that

$$
H^{p}\left(\hat{W}_{n}, \mathscr{S}_{\varepsilon} L\right)=0 \quad \text { for } \quad n, p \geqslant 1 .
$$

Let

$$
0 \rightarrow \mathscr{S}_{\varepsilon} L \rightarrow J_{0} \stackrel{d_{0}}{\rightarrow} J_{1} \stackrel{a_{1}}{\rightarrow} \ldots
$$

be a flabby resolution of $\mathscr{S}_{\varepsilon} L$. Since

$$
0 \rightarrow \mathscr{S}_{\varepsilon} L\left|\hat{W}_{n} \rightarrow J_{0}\right| \hat{W}_{n} \rightarrow J_{1} \mid \hat{W}_{n} \rightarrow \ldots
$$

is a flabby resolution of $\mathscr{S}_{\varepsilon} L \mid \hat{W}_{n}$, by (1.5) we have

$$
\widehat{\operatorname{Ker} d_{p} \mid \hat{W}_{n}}=\widehat{\operatorname{Im} d_{p-1}} \mid \hat{W}_{n} \quad \text { for } \quad n, p \geqslant 1 .
$$

Let $\sigma \in \widehat{\operatorname{Ker} d_{q}}$. By (1.6) for every $n \geqslant 1$ there exists an $a^{(n)} \in H^{0}\left(\hat{W}_{n}, J_{q-1}\right)$

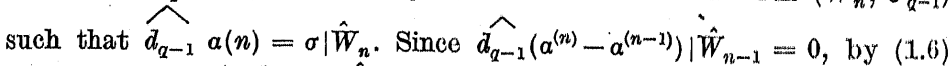
there exists a $\beta^{(n-1)} \in H^{0}\left(\hat{W}_{n-1}, J_{q-2}\right)$ such that

$$
\widehat{a_{\alpha-2}} \beta^{(n-1)}=\left(a^{(n)}-\alpha^{(n-1)}\right) \mid \hat{W}_{n-1} .
$$

Since $J_{p}$ are flabby for every $n$, there exists an $\tilde{\beta}^{(n-1)} \in J_{q-2}(X)$ such that $\tilde{\beta}^{(n-1)} \mid \hat{W}_{n-1}=\beta^{(n-1)}$. Define an $\alpha \in J_{q-1}(X)$ by

$$
a\left|W_{n}=\left(\alpha^{(n)}-\widehat{d_{q-2}}\left(\sum_{k<n} \tilde{\beta}^{(k)}\right)\right)\right| W_{n}
$$

This is well defined since for $n \geqslant m$ we have

$$
\begin{aligned}
a^{(n)}-\widehat{d_{\alpha-2}}\left(\sum_{k<n} \tilde{\beta}^{(k)}\right)-\left(\alpha^{(m)}-\widehat{d_{q-2}}\left(\sum_{k<m} \tilde{\beta}^{(k)}\right)\right. \\
\quad=a^{(n)}-\alpha^{(m)}-\widehat{d_{q-2}}\left(\sum_{m<k<n} \tilde{\beta}^{(k)}\right) \\
=a^{(n)}-\alpha^{(m)}-\sum_{m \varepsilon k<n}\left(a^{(k+1)}-\alpha^{(k)}\right)=0 \text { on } W_{n} .
\end{aligned}
$$

Finally on $W_{n}, \hat{a_{\alpha^{-1} 1}} \alpha=\sigma \mid W_{n}$. Thus $\widehat{a_{\alpha-1}} \alpha=\sigma$.

OoromitakX 1.10. Oousin's First Problem for holomorphic functions on a Stein space with values in $T^{\prime}$, where $F$ is a Fréchet space which does not admit a continuous norm, may not admit a solution.

It is known [2] that Cousin's First Problem for holomorphic functions on a Stein space with values in a Fréchet space has a solution.

Now we apply Theorems 1.1 and 1.2 to get the splitness of Dolbeault complexes of holomorphic vector bundles over Stein manifolds at only positive dimension. This result has been by Palamodor [8]. We need the following

LFima 1.11. Let $\triangle$ be a oompact polydiso in $C^{n}$ and $\omega$ a $0^{\infty}$-form of bidegree $(p, q)$ on a neighbourhood of $\bar{\Delta}$ with values in a quasi-complete looally oonveso space $L$. If $q>0$ and $\bar{\partial}_{L}^{a} \omega=0$, where $\bar{\partial}_{L}^{\alpha}=\bar{\partial}^{q} \varepsilon$ id, then there exists a $\theta^{\infty}$-form $\eta$ of bidegree $(p, q-1)$ on a neighbourhood of $\triangle$ with values in $x$ such that $\bar{\partial}_{L}^{\alpha-1} \eta=\omega$.

Proof. For $L=C$ the lemma has been established in [6]. Let us notice that the proof in [6] gives also a proof of Lemma 1.10 since we have the following

Romark 1.12. Let $D$ be a simple connected domain in $\boldsymbol{C}$ and $g$ a $0^{\infty}$-function on $D$ with values in a quasi-complete locally convex space $I$ such that suppg is compact. Then setting

$$
f(z)=1 / 2 \pi i \iint_{D} \frac{g(\xi) d \xi \wedge d \xi}{\xi-z}
$$

for every $\approx \in D$ we get w $\mathcal{O}^{\infty}$-function $f$ on $D$ with values in $L$ so that $\bar{\partial}_{x}^{0} f=g$. Morcover, if $g$ is $C^{\infty}$ or holomorphic in some additional parameters, so is $f$.

Let $\xi$ bo a holomorphic Banach bundle over a complex manifold $X$. By $O_{\xi}$ wo denote the sheaf of germs of holomorphic sections of $\xi$ on $X$ and by $\Omega^{(p, q)}$ the sheat of germs of $O^{\infty}$-forms of bidegree $(p, q)$ on $X$ with values in $\xi$. Lemma 1.11 implies that the sequence 
is exact, where $\Omega_{\xi}^{q}=\Omega_{\xi}^{(0, q)}$. The complex.

$$
D(\xi): 0 \rightarrow \mathcal{O}_{\xi}(X) \rightarrow \Omega_{\xi}^{0}(X) \stackrel{\hat{\partial}_{\xi}^{0}}{\rightarrow} \Omega_{\xi}^{1}(X) \stackrel{\frac{\hat{\partial}_{\xi}^{1}}{\rightarrow}}{\rightarrow} \ldots
$$

of global sections of (1.7) on $X$ is called the Dolbeault complex of $\xi$. We say that the complex $D(\xi)$ splits at $q$ if there exists a continuous lincur

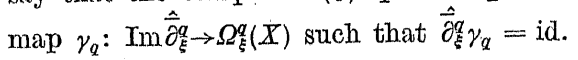

Proposrtron 1.13. Let $\xi$ be a holomorphio vector bundle on a stein manifold $X$. Then $D(\xi)$ splits at $q$ if and only if $q>0$.

Proof. Lemma 1.11 implies that for every quasi-complote locally convex space $L$ the sequence

$$
0 \rightarrow \mathcal{O}_{\xi} \varepsilon L \rightarrow \Omega_{\xi}^{0} \varepsilon L \rightarrow \Omega_{\xi}^{1} \varepsilon L \rightarrow \ldots
$$

which is obtained by tensoring the sequence (1.7) with $L$ is exact. Hence

$$
\begin{aligned}
H^{q}\left(X, \mathcal{O}_{\xi} \varepsilon L\right) & =\operatorname{Ker}\left(\bar{\partial}_{\xi}^{q} \varepsilon \mathrm{id}\right)^{\wedge} /\left(\operatorname{Im} \bar{\partial}_{\xi}^{q} \varepsilon \mathrm{id}\right)^{\wedge} \\
& =\operatorname{Ker} \hat{\bar{\partial}} \bar{\xi} \varepsilon L / \operatorname{Im}\left(\bar{\partial}_{\xi}^{q-1} \varepsilon \mathrm{id}\right)^{\wedge} .
\end{aligned}
$$

Let $q>0$. Applying Theorem 1.2 (ii) to $L=\left(\operatorname{Im} \hat{\bar{\partial}}_{\xi}^{q}\right)^{\prime}$, by (1.9) it follows that the $\operatorname{map}\left(\bar{\partial}_{\xi}^{q} \varepsilon \mathrm{id}\right)^{\wedge}: \Omega_{\xi}^{q}(X) \varepsilon\left(\operatorname{Im} \hat{\bar{\partial}}_{\xi}^{q}\right)^{\prime} \rightarrow\left(\operatorname{Im} \hat{\overline{\partial_{\xi}}}\right) \varepsilon\left(\operatorname{Im} \hat{\bar{\partial}}_{\xi}^{q}\right)^{\prime}$ is surjective. This implies that $D(\xi)$ splits at $q$.

Now we prove that $D(\xi)$ does not split.at 0 . For a contradiction. by (1.9) it follows that

$$
H^{1}\left(Z, \mathcal{O}_{\xi} \varepsilon L\right)=0
$$

for every quasi-complete locally convex space $L$, where $Z$ is some component of $X$. By Theorem 1.1 (i) the space $\mathcal{O}_{\xi}(Z)$ is isomorphic to $C^{m}$ for $m \leqslant \infty$. This is imposible, since $\mathcal{O}_{\xi}(Z)$ is an infinite-dimonsional Fréchet space having a continuous norm. The proposition is proved.

\section{Splitness of Dolbeault complexes of holomorphic Banach bundles.} Let $X$ be an analytic space. We say that $X$ has a Stein morphism if there exists a holomorphic map $\pi$ from $X$ into a Stcin space $W$ such that $\pi^{-1}(U)$ is Stein for every Stein open set $U$ belonging to a stein open covoring $\mathscr{U}$ of $W$. Since $\pi\left(\partial \pi^{-1}(V)\right) \subseteq \partial V$ for every open sot $V \subset W$ it is easy to see that $\pi^{-1}(V)$ is Stein for every Stcin opon set $V$ in $X$ contained in some $U \in \mathscr{U}$. Thus by a lemma of Stehle [11] there exists a Stein open covering $\left\{\nabla_{j}\right\}_{j=1}^{\infty}$ of $W$ such that

$\left(\mathrm{S}_{1}\right) \quad \Omega_{j}=\bigcup_{i \leqslant j} V_{i}$ are Stein.

$\left(\mathrm{S}_{2}\right) \quad \Omega_{j} \cap V_{j+1}$ are Runger domains in $V_{j+1}$.

$\left(\mathrm{S}_{8}\right) \quad \tilde{\mathscr{U}}=\left\{\boldsymbol{X}_{j}^{\prime}=\pi^{-1}\left(\nabla_{j}\right)\right\}$ is a Stein covering of $\boldsymbol{X}$.
Since $X_{j+1}^{\prime}$ is Stein, by $\left(\mathrm{S}_{2}\right)$ it is easy to check that $X_{j} \cap X_{j+1}^{\prime}$ is Runger in $X_{j+1}^{\prime}$.

In this section we prove the following

TrEOREM 2.1. Let $\xi$ be a holomorphio Banach bundle over a Stein manifold $X$. Then $D(\xi)$ splits at $q$ if and only if $q>0$.

TheоRIM 2.2. Let $\xi$ be a holomorphic Banach bundle over a complex manifold $X$ having a Stein morphism. Then

(i) $D(\xi)$ splits at $q \geqslant 2$.

(ii) $D(\xi)$ does not split at 0 .

Proof of Theorem 2.1. We can assume that $X$ is connected. First wo prove that $D(\xi)$ does not split at 0 . For a contradiction by (1.9) we have

$$
H^{1}\left(X, \mathcal{O}_{\xi} 8 C^{\infty \prime}\right)=0 .
$$

Select $\sigma \in \mathcal{O}_{\xi}(X), \sigma \neq 0$. Since $X$ is Stein, there exists a sequence $\left\{z_{n}\right\} \subset X$ and $f \in \mathcal{O}(X)$ such that

$\sigma\left(z_{j}\right) \neq 0$ for $j \geqslant 1$,

$$
\left|f\left(z_{j}\right)\right| \rightarrow \infty \text { and } f\left(z_{j}\right) \neq f\left(z_{k}\right) \text { for } j \neq k \text {. }
$$

Consider the function $\varphi \in \mathcal{O}(C)$ and the covering $\mathscr{U}$ as in the proof of Thoorem 1.2 (i). Put

$$
\sigma_{i j}=0 \text { for } i, j \geqslant 1 \text { and } \sigma_{0 j}=-\sigma_{j 0}=\sigma / \varphi f \otimes e_{j} \quad \text { for } j \geqslant 0 .
$$

Obviously $\left\{\sigma_{i j}\right\} \in Z^{1}\left(\mathscr{U}, \mathscr{O}_{\xi} \varepsilon C^{\left(\alpha^{\prime}\right.}\right)$. Hence by (2.1) we can assume that

whonces

$$
\sigma_{i j}=\sigma_{i}-\sigma_{j}, \quad \text { where } \quad \sigma_{j} \in H^{0}\left(U_{j}, \mathcal{O}_{\xi} \varepsilon C^{\infty \prime}\right) \text {, }
$$

$$
\varphi f \sigma_{i}+\sigma \otimes e_{i}=\varphi f \sigma_{j}+\sigma \otimes e_{j} \quad \text { on } \quad U_{i} \cap U_{j} .
$$

Thus there exists an element $\beta \in H^{0}\left(X, \mathcal{O}_{\xi} \varepsilon C^{\infty \prime \prime}\right)$ such that $\beta\left(z_{j}\right)=\ddot{\sigma}\left(z_{j}\right) \otimes e_{j}$ for $j \geqslant 1$. By the connectedness of $X$ and since $H^{0}\left(X, O_{\xi} \varepsilon C^{\infty \prime}\right)=H^{0}(X$, $\left.\mathcal{O}_{\varepsilon}\right){ }_{8} C^{\circ 0 \prime}$ it follows that

$$
\beta \in H^{0}\left(H, \mathcal{O}_{\xi}\right) e_{1} \oplus \ldots \oplus H^{0}\left(X, \mathcal{O}_{\xi}\right) e_{n_{0}} \quad \text { for some } n_{0} .
$$

This contradictis the rolation

$$
\beta\left(z_{n_{0}+1}\right)=\sigma\left(z_{n_{0}+1}\right) e_{n_{0}+1} \notin H^{0}\left(X, \mathcal{O}_{\xi}\right) e_{1} \oplus \ldots \oplus H^{0}\left(X, \mathcal{O}_{\xi}\right) e_{n_{0}}
$$

since $\sigma\left(z_{n_{0}+1}\right) \neq 0$.

Now let $q>0$. Wo provo that $D(\xi)$ splits at $q$.

(a) Tixst wo prove that

(2.2) $H^{p}\left(X, \mathcal{O}_{\xi} \varepsilon L\right)=0$ for every $p \geqslant 2$ and for overy quasi-complete locally convex space $L$. 
By the proof of Theorem 1.2 (ii) it suffices to prove that

$$
H^{p}\left(\hat{W}, \mathcal{O}_{\xi} \varepsilon L\right)=0
$$

for every compact subset $W$ of $X$ and every $p \geqslant 1$. Since the relation

$$
\bigcup\{B \varepsilon L(K): K \in \mathscr{B}(L)\}=\bigcup\{[B \varepsilon L](K): K \in \mathscr{B}(B \varepsilon L)\}
$$

holds for all Banach spaces $B$, it follows that

$$
\left(\mathcal{O}_{\xi} \varepsilon L\right)_{z}=\bigcup\left\{\left(\mathcal{O}_{\xi} \varepsilon L(K)\right)_{z}: K \in \mathscr{B}(L)\right\} \quad \text { for } \quad z \in X .
$$

Thus

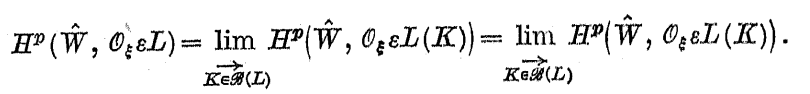

Since $\hat{W}$ has a basis of Stein neighbourhoods, we infer that

$$
H^{p}\left(\hat{W}, \mathcal{O}_{\xi} \varepsilon L\right)=\lim _{\substack{G \rightarrow \hat{W} \\ G \text { is Stein }}} \lim _{\overrightarrow{K \in \mathscr{P}}(L)} H^{p}\left(G, \mathcal{O}_{\xi} \varepsilon L(K)\right)=0 .
$$

(b) Using $(2.2)$ to $L=\left(\operatorname{Im} \hat{\hat{\partial}_{\xi}^{q}}\right)_{c}^{\prime}$, by the relation $\left(\left(\operatorname{Im} \hat{\bar{\partial}}_{\xi}^{q}\right)_{c}^{\prime}\right)_{c}^{\prime}=\operatorname{Im} \hat{\hat{\partial}_{\tilde{\xi}}^{q}}$, we infer that the map

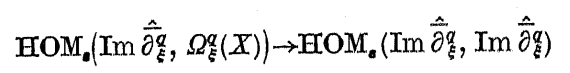

induced by

$$
\hat{\bar{\partial}} \frac{q}{\xi}: \Omega_{\xi}^{q}(X) \rightarrow \operatorname{Im} \hat{\bar{\partial}} q
$$

is surjective. This implies that $D(\xi)$ splits at $q$. The theorem is proved. The proof of Theorem 2.2 is based on the following

Proposimion 2.3. Let $\xi$ and $X$ be as in Theorem 2.2 and let $z_{0} \in X$. Let $J_{\xi z_{0}}$ denote the subsheaf of the sheaf $\mathcal{O}_{\xi}$ consisting of germs which vanish at $z_{0}$. Then

(i) there exists a Stein open covering $\tilde{\mathscr{U}}_{1}$ of $X$ such that the map $\delta^{0}$ : $C^{0}\left(\tilde{\mathscr{U}}_{1}, J_{\xi \varepsilon_{0}}\right) \rightarrow Z^{\mathbb{1}}\left(\tilde{\mathscr{U}}_{1}, J_{\xi \Sigma_{0}}\right)$ has dense image.

(ii) $H^{Q}\left(X, \mathcal{O}_{\xi} \varepsilon L\right)=0$ for every $q \geqslant 3$ and for every quasi-complete locally convex space $L$.

Proof. (i): We write $\mathscr{S}=J_{\xi \tilde{z}_{0}}$. Take a Stein open covering $\tilde{\mathscr{U}}_{1}$ of $X$ such that $\tilde{\mathscr{U}}_{1}<\tilde{\mathscr{U}}, \tilde{\mathscr{U}}_{1}$ forms a basis of open sets in $X$ and

$$
\tilde{\mathscr{U}}_{1}\left|X_{j+1}=\tilde{\mathscr{U}}_{1}\right| X_{j} \cup \tilde{\mathscr{U}}_{1} \mid X_{j+1}^{\prime}
$$

for $j \geqslant 1$, where $\tilde{\mathscr{U}}_{1} \mid G=\left\{U \in \tilde{\mathscr{U}}_{1}: U \subset G\right\}$. We prove that $\operatorname{Im} \delta^{0}\left(\tilde{\mathscr{U}}_{1}, \mathscr{S}\right)$ is dense in $Z^{1}\left(\tilde{\mathscr{U}}_{1}, \mathscr{S}\right)$. It suffices to show that

$$
\overline{\operatorname{Im} \delta^{0}\left(\tilde{\mathscr{U}}_{1} \mid X_{j}, \mathscr{S}\right)}=Z^{1}\left(\tilde{\mathscr{U}}_{1} \mid X_{j}, \mathscr{S}\right) \quad \text { for } \quad j \geqslant 1
$$

Since $\tilde{U}_{1} \mid X_{1}$ is a Leray covering of the Stein manifold $X_{1},(2.4)$ holds for $j=1$.

Now given $\varphi \in Z^{1}\left(\tilde{\mathscr{U}}_{1} \mid X_{j+1}, \mathscr{S}\right)$. Take a sequence $\left\{\alpha_{n}^{j}\right\} \subset \mathscr{V}^{0}\left(\tilde{\mathscr{U}}_{1} \mid X_{j}, \mathscr{P}\right)$ such that $\delta^{0} \alpha_{n}^{j} \rightarrow \varphi\left|\tilde{U}_{1}\right| X_{j}$. Since $\left(\delta^{0} \alpha_{n}^{j}-\varphi\right)\left|\tilde{U}_{1}\right| X_{j} \cap X_{j+1}^{\prime} \rightarrow 0$ and the map

$\varrho: A=\sigma^{0}\left(\tilde{\mathscr{U}}_{1} \mid X_{j}, \mathscr{S}\right) \oplus \sigma^{0}\left(\tilde{\mathscr{U}}_{1} \mid X_{j} \cap X_{j+1}^{\prime}, \mathscr{S}\right) \rightarrow Z^{1}\left(\tilde{\mathscr{U}}_{1} \mid X_{j} \cap X_{j+1}^{\prime}, \mathscr{S}\right)$

dofined by

$$
\varrho(\sigma, \beta)=\delta^{0}(\sigma-\beta)\left|\tilde{U}_{1}\right| X_{j} \cap X_{j+1}^{\prime}
$$

is surjective, there exists a sequence $\left\{\left(\gamma_{n}^{1}, \gamma_{n}^{2}\right)\right\} \subset A$ converging to zero such that

$$
\begin{aligned}
\varrho\left(\left(\gamma_{n}^{1}, \gamma_{n}^{2}\right)\right) & =\delta^{0} \alpha_{n}^{j}-\varphi\left|\tilde{\mathscr{U}}_{1}\right| X_{j} \cap X_{j+1}^{\prime} \\
& =\delta^{0} \alpha_{n}^{j}\left|\tilde{\mathscr{U}}_{1}\right| X_{j} \cap \bar{X}_{j+1}^{\prime}-\delta^{0} \beta\left|\tilde{\mathscr{U}}_{1}\right| X_{j} \cap X_{j+1}^{\prime},
\end{aligned}
$$

where $\beta \in \mathcal{O}^{0}\left(\tilde{\mathscr{U}}_{1} \mid X_{j+1}^{\prime}, \mathscr{S}\right), \delta^{0} \beta=p \mid X_{j+1}^{\prime}$.

Since $X_{i} \cap X_{j+1}^{\prime}$ is a Runger domain in $X_{j+1}^{\prime}$, there exists a sequence $\left\{h_{n}\right\} \subset H^{0}\left(X_{j+1}^{\prime}, \mathscr{S}\right)$ such that $u_{n}-h_{n} \rightarrow 0$ on $X_{j} \cap X_{j+1}^{\prime}$, where $u_{n}=\left(a_{n}^{j}-\right.$ $\left.-\gamma_{n}^{1}\right)-\left(\beta-\gamma_{n}^{2}\right) \in H^{0}\left(X_{j} \cap X_{j+1}^{\prime}, \mathscr{S}\right)$. Let $\tilde{u}_{n}$ and $\tilde{h}_{n}$ be trivial extensions of $u_{n}$ and $h_{n}$, repectively, on $X_{j}$. Put

$$
\alpha_{n}^{j+1}=\left\{\begin{array}{lll}
\alpha_{n}^{j}-\gamma_{n}^{1}-\tilde{u}_{n}-\tilde{h}_{n} & \text { on } & \tilde{\mathscr{U}}_{1} \mid X_{j}, \\
\beta-\gamma_{n}^{2}-h_{n} & \text { on } & \tilde{\mathscr{U}}_{1} \mid X_{j+1}^{\prime}
\end{array}\right.
$$

Then $\left\{\alpha_{n}^{j+1}\right\} \subset \mathscr{O}^{0}\left(\tilde{\mathscr{U}}_{1} \mid X_{j+1}, \mathscr{S}\right)$ and $\delta^{0} \alpha_{n}^{j+1} \rightarrow \varphi\left|\tilde{\mathscr{C}}_{1}\right| X_{j+1}$.

(ii): (a) Since $H^{\alpha}\left(Z, \mathcal{O}_{\xi} \varepsilon L\right)=0$ for every $q \geqslant 2$ and for every Stein open set $Z$ in $X$, by induction on $j$ and considering the Mayer-Vietoris sequence of pairs $\left(X_{j}, X_{j+1}^{\prime}\right)$ we infer that

$$
H^{\alpha}\left(X_{j}, \mathscr{O}_{\xi} \varepsilon L\right)=0 \text { for } q \geqslant 3 \text { and for } j \geqslant 1 \text {. }
$$

(b) Take a flabby resolution

$$
0 \rightarrow \mathcal{O}_{\xi} \varepsilon L \rightarrow J_{0} \stackrel{d_{0}}{\rightarrow} J_{1} \stackrel{a_{1}}{\rightarrow} \ldots
$$

of the sheaf $\mathcal{O}_{\xi} \varepsilon$. We prove that for every $j \geqslant 1$ and for every $q \geqslant 3$ the restriction map $\widehat{\operatorname{Ker} d_{q-1}}\left|X_{j+1} \rightarrow \widehat{\operatorname{Ker} d_{q-1}}\right| X_{j}$ is surjective. Given $\alpha \in{\widehat{K K C r} d_{a-1}}_{1} \mid X_{j}$. Since $X_{j} \cap X_{j+1}^{\prime}$ is Stein and $q-1 \geqslant 2$, by (2.2) we find $\eta \in J_{\alpha-1}\left(X_{j} \cap X_{j+1}^{\prime}\right)$ such that $\widehat{d_{\alpha-1}}\left|X_{j} \cap X_{j+1}^{\prime} \eta=\alpha\right| X_{j} \cap X_{j+1}^{\prime}$. Let $\tilde{\eta}$ be an oxtension of $\eta$ on $X_{j+1-1}^{\prime}$. Setting

$$
\tilde{\alpha} \mid X_{j}=\alpha \quad \text { and } \quad \tilde{\alpha}\left|X_{j+1}^{\prime}=\widehat{d_{\alpha-2}}\right| X_{j+1}^{\prime} \tilde{\eta},
$$

wo get an eloment $\tilde{a} \in \operatorname{Ker} d_{\alpha-1} \mid X_{j+1}^{\prime}$ extending $\alpha$.

(c) Let $a \in \operatorname{Krcr} \widehat{d_{\alpha}}, q \geqslant 3$. Talke $a_{1} \in J_{q-1}\left(X_{1}\right)$ such that $\widehat{d_{q-1}} \mid X_{1} a_{1}$ 
$=\alpha \mid X_{1}$. By (2.5) there exists $a_{2}^{\prime} \in J_{q-1}\left(X_{2}\right)$ such that $\widehat{d_{q-1}} a_{2}^{\prime}=\alpha \mid X_{2}$. Since $\left(a_{2}^{\prime}-\alpha_{1}\right)\left|X_{1} \in \operatorname{Ker} \widehat{d_{q-1}}\right| X_{1}$, by (b) we find $\alpha_{2}^{\prime \prime} \in \operatorname{Ker} \widehat{d_{q-1}} \widehat{X_{2}}$ extending $\left(\alpha_{2}^{\prime}-a_{1}\right) \mid X_{1}$. Put $\alpha_{2}=\alpha_{2}^{\prime}-\alpha_{2}^{\prime \prime}$. Then $\alpha_{2} \in J_{q-1}\left(X_{2}\right)$ and

$$
a_{2}\left|X_{1}=a_{1}, \quad \widehat{d_{q-1}}\right| X_{2} \alpha_{2}=\alpha \mid X_{2} .
$$

Continuing this process we get elements $\alpha_{n} \in J_{q-1}\left(X_{n}\right)$ such that

$$
a_{n} \mid X_{n-1}=a_{n-1} \text { and } \widehat{d_{q-1}}\left|X_{n} a_{n}=\alpha\right| X_{n} \text { for } n \geqslant 2 .
$$

Thus the formula $\beta \mid X_{n}=a_{n}$ for $n \geqslant 1$ defines an element $\beta \in J_{q-1}(X)$ such that $\widehat{d_{q-1}} \beta=\alpha$. Hence (ii) is proved.

The proof of (i) is similar to a proof of Jennane [7].

Proof of Theorem 2.2. The splitness of $D(\xi)$ at $q \geqslant 2$ follows from Proposition 2.3 (ii) and from the proof of Theorem 2.1.

Now we prove that $D(\xi)$ does not split at 0 . For a contradiction by Proposition 2.3 (i) we have $H^{1}\left(X, \mathcal{O}_{\xi}\right)=0$. Hence, by the splitness of $D(\xi)$, we infer that (2.1) holds. By (2.1) and by the proof of Theorem. 2.1 it suffices to show that $\mathcal{O}_{\xi}(X) \neq 0$ and $\operatorname{dim} \mathcal{O}(X)>1$.

Obviously $\operatorname{dim} \mathscr{O}(X)>1$ since $X$ has a Stein morphism.

Assume that $\mathcal{O}_{\xi}(X)=0$. Take $z_{0} \in X$ such that $\xi_{z_{0}} \neq 0$. Let $\tilde{\mathscr{U}}_{1}$ and $\mathscr{S}^{\prime}=J_{\xi z_{0}}$ be as in Proposition 2.3. Then $H^{1}\left(X, \mathscr{S}^{\prime}\right)=Z^{0}\left(\tilde{\mathscr{U}}_{1}, \mathscr{S}^{\prime}\right) / \operatorname{Im} \delta^{0}\left(\tilde{\mathscr{U}}_{1}\right.$, $\left.\mathscr{S}^{\prime}\right)$ and $\operatorname{Im} \delta^{0}\left(\tilde{\mathscr{U}}_{1}, \mathscr{S}^{\prime}\right)$ is dense in $Z^{1}\left(\tilde{\mathscr{U}}_{1}, \mathscr{S}^{\prime}\right)$. Then, by the exactness of the following commutative diagram

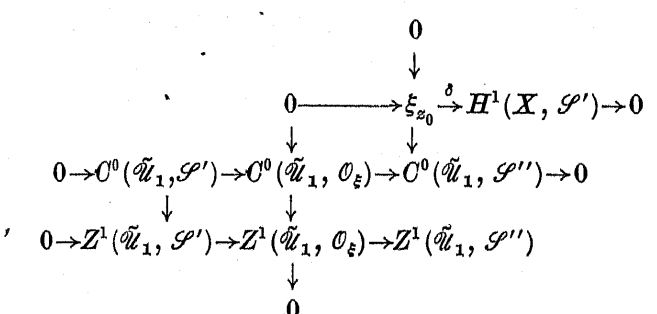

we infer that $\operatorname{Im} \delta^{0}\left(\tilde{\mathscr{U}}_{1}, \mathscr{S}\right)$ is closed and hence $\mathscr{H}^{1}(X, \mathscr{S})=0$. This implies that $\xi_{z_{0}}=0$ which contradicts choice of $z_{0}$. The theorem is proved.

\section{References}

[1] C. Bessaga and A. Pełczyński, On a olass of $B$ spaces, Bull. Acad. Polon. Sci. 5 (1957), 375-379.

[2] L. Bungart, Holomorphic functions with values in locally convex spaces and applications to integral formula, Trans. Amer. Math. Soc. 111 (1964), 317-343.
[3] - On analytio fiber bundles. I. Holomorphic fiber bundles with infinite dimensional fiber, Topology 7 1 (1968), 55-68.

[4] A. M. Gloasion, The abstract theorem of Oauchy-Weil, Pacific J. Math. 12. 2 (1962), $511-526$. [5] 'R. Godemont, Topologie algebrique et théorie de faisceaux, Hermann, Paris
1958.

[6] R. Gunning and H. Rossi, Analytio Functions of Several Oomplex.

[7] B. Jonnuno, Groupes de cohomologie d'un fibré holomorphe à base et fibre do Stein, Śminaire Lilong, Skoda, Lucture Notes 822 (1978/79), 100-108.

[8] P. V. Palamodov, On Stein manifolds Dolbeaut complexes splits at positive dimension, Mat. Sb. 88 (1972), 287-315.

[9] A. Pistisoh, Nuolear Looally Oonvex Spaces, Akademie-Verlag, Berlin 1972 [10] It. Schaf'ir, T'opologioal Veotor Spaces, Now York 1966.

[11] J. L. S tolhé, Fonotions plurisousharmoniques et convexité holomorphe de certains fibrés analytiques, Séminaire Lelong, Lecture Notes 474 (1973/74), 155-179.

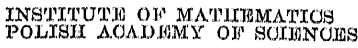

